\title{
Guided Sentences Composition for Disabled People
}

\author{
Robert Pasero Nathalie Richardet Paul Sabatier \\ Laboratoire d'Informatique de Marseille CNRS URA 1787 \\ Faculté des Sciences de Luminy 163 Avenue de Luminy - Case 901 \\ 13288 Marseille Cedex 9 - France \\ \{Robert-Pasero,Nathalie-Richardet,Paul-Sabatier\}@gia.univ-mrs.fr
}

\begin{abstract}
We present the advantages of guided sentences composition for communicating in natural language with computers. We show how guidance can be achieved by means of the partial synthesis of sentences and describe our approach consisting in separating knowledge that comes under different levels of well-formedness and coroutining their treatment. This approach has led us to develop in Prolog a software, ILLICO, allowing for the conception of natural language interfaces with guided sentences composition. We present an application we have developed by means of ILLICO : KOMBE, a communication aid system for handicapped persons.
\end{abstract}

\section{Guided Sentences Composition}

We may distinguish two kinds of communication with natural language interfaces :

- a "free" mode : the user types sentences without knowing the limits of the interface but he hopes he will be understood. Trivial reality : user's freedom will always be the freedom the system will grant him.

- a guided mode : the system guides the user while he composes sentences.

Unlike the "free" mode, with guided composition users quickly perceive the limits of the interface. The designer does not have to program all the expressions or structures having the same meaning. Unique forms and structures are sufficient. He may forget the others. Guided composition is a powerful principle for natural language interfaces to database and knowledge access, operating and command systems. This principle can also be applied to learning systems (natural languages or formal ones like database or programming languages).

Expected formulations can be provided to the user by means of partial synthesis of sentences. This principle was introduced by Alain Colmerauer within the French-English interface, Orbis, which was connected to a database on planets (Colmerauer and Kittredge, 1982). The same system is used for analyzing a given sentence and for synthesizing expected fomulations. Orbis is implemented in Prolog and illustrates in a convincing manner the potential reversibility of certain programs written in that language.
We are concerned here with the partial synthesis of sentence from left to right. The main problem of this approach is that a word or an expression that has been synthesized by the system (and selected by the user to compose his sentence) must not lead to a future dead end. Theoretically, dead ends may issue from different levels corresponding to the differents levels of well-formedness of a sentence : lexical, syntactic, conceptual and contextual levels, in particular.

As a result of formal language theory, we know that the emptiness problem is solvable for context-free grammars. So one can take all the levels of well-formedness into account simultaneously by merging them into one formalism, a context-free grammar with symbols reflecting lexical, syntactic, conceptual and contextual constraints, as it is done for instance in "semantic grammars". This is the case in Tennant's menu-based system (Tennant, 1984).

The approach we have decided to follow is different. We are interested in (1) separating knowledge that comes under different levels (lexical, syntactic, conceptual) into distinct modules and (2) coroutining their treatment in order to avoid dead ends along the guided composition mode.

\section{ILLICO}

In our approach, knowledge is separated into four distinct modules :

- a set of lexical rules (the lexicon) specifies expected words and expressions ;

- in a restricted version of the Metamorphosis Grammar formalism (Colmerauer, 1975), a set of syntactic rules (the grammar) defines expected structures of sentences and grammatical agreements ;

- by means of lambda-expressions operating on the semantic representation associated with relevant syntactic categories, a set of semantic rules allows the production of semantic representations ;

- a set of conceptual rules (the conceptual model) defines domain constraints and connectivity constraints (Godbert et al., 1993).

The algorithm for coroutining the treatment of the different kinds of knowledge in order to avoid dead ends along the guided composition mode is described in (Milhaud et al., 1992).

Our approach has led us to develop in Prolog a software, ILLICO, allowing for the conception of natural language interfaces with guided sentences 
composition. The following figure illustrates the general principle of the approach.

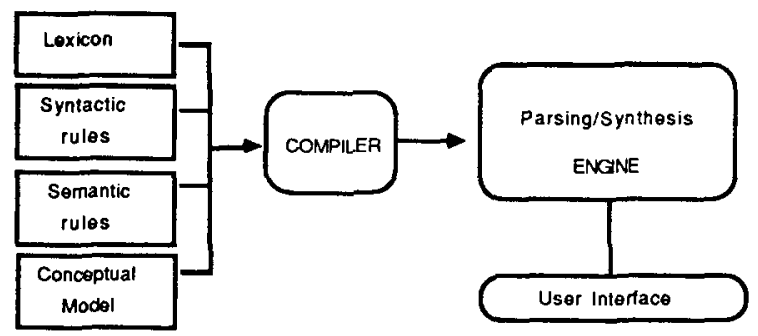

4 The KOMBE System

In the framework of the EC TIDE program, we have developped by means of ILLICO, a communication aid system for disabled people : the KOMBE system. KOMBE is devoted to Amyothrophic Lateral Sclerosis (ALS) patients (Guenthner et al., 1992). In addition to motor weaknesses, ALS patients, mainly elderly people, suffer from diseases of the central nervous system that entail increasing speech impairment and aggravate their living conditions. They have not lost their cognitive capacities but are unable to speek and some of them are unable to write and communicate only by eye movements. For this kind of disabled person, the computer-assisted aids developed so far offer the user relatively slow communication, composing sentences letter by letter or selecting a pre-formulated phrase or sentence. Some systems include a dictionary permitting the user to select whole words. Guided sentences composition is a more convivial way for them to communicate. Step by step, they can select on the screen for selecting words and expression dynamically synthesized by the system. Words and expressions generated by the system always lead to the construction of a lexically, syntactically and conceptually well-formed sentence. The following figures show an example of a partial composition of a sentence by an ALS patient communicating with a doctor.

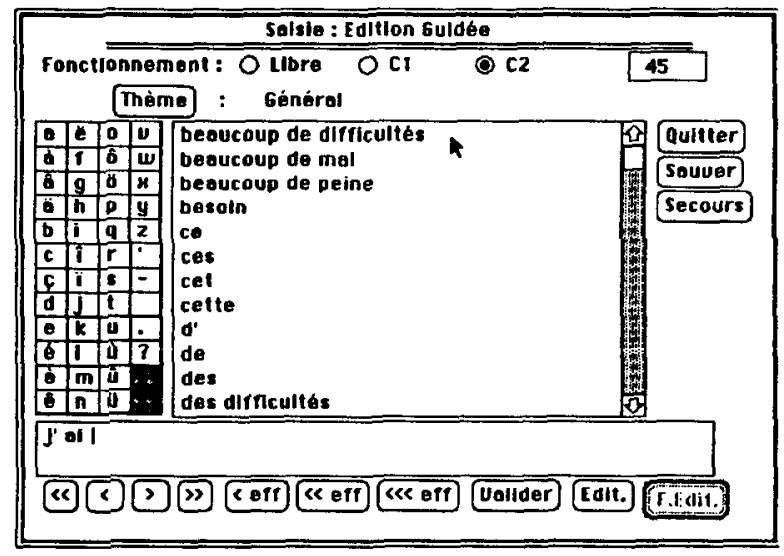

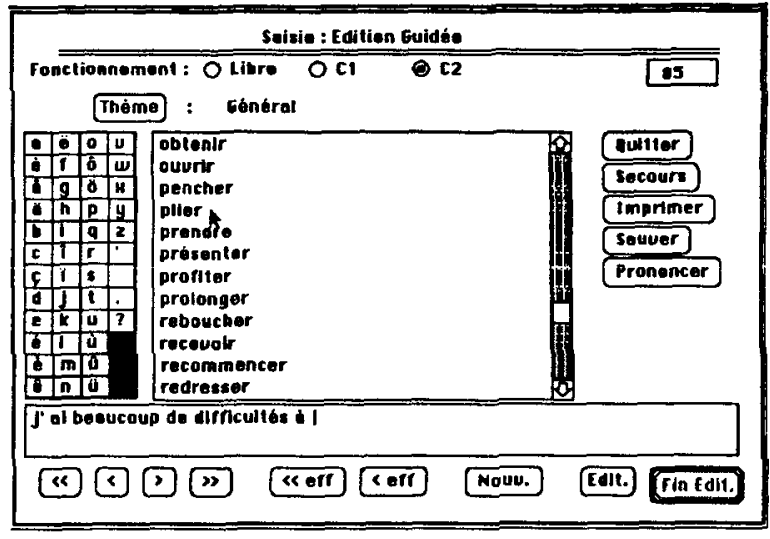

For example, the patient can compose the sentence : J'ai beaucoup de peine à plier le genou gauche (It is difficult for me to bend my left knee).ALS patients can compose sentences about several conceptual situations. The introduction of a new situation only needs the definition of the associated conceptual model and lexicon. The grammar and the other components of the system are domain independant.

In order to resolve the big lexicon problem, the system proposes several "sub-lexicon" of non empty intersection. This thematic division is built on tree structure and operates on the lexicon level.

\section{Acknowledgments}

Parts of this work were funded by the French Ministère de la Recherche et de la Technologie (ILLICO Project) and the EC TIDE (Technology Initiative for Disabled and Elderly people) program (KOMBE Project). Special thanks to Franz Guenthner and Karin Krüger-Thielmann for their contributions in the KOMBE Project.

\section{References}

Colmerauer A. 1975. Metamorphosis Grammars. Natural Language Communication With Computers, Springer Verlag.

Colmerauer A. and Kittredge R. 1982. ORBIS : Proceedings of the 9 th COLING Conference.

Godbert E., Pasero R. and Sabatier P. 1993. Specifying and Using Conceptual Constraints. Proceedings of the Fifth International Conference on Human-Computer Interaction, Elsevier.

Guenthner F., Krüger-Thielmann K., Pasero R. and Sabatier P. 1992. Communications Aids for ALS Patients. Proceedings of the 3rd International Conference on Computers for Handicapped Persons.

Milhaud G., Pasero R. et Sabatier P. 1992. Partial Synthesis of Sentences by Coroutining Constraints on Differents Levels of WellFormedness. Proceedings of the 14th COLING Conference.

Tennant H. 1984. Menu-Based Natural Language Understanding. Proceedings of the National Computer Conference. 